\title{
Ausência da atividade anti-helmíntica de plantas em frangos de corte naturalmente infectados com Heterakis gallinarum (Schranck,1788) Madsen,1949
}

\author{
Absence of the anthelminthic activity of plants in cut chickens naturally infected with Heterakis \\ gallinarum (Schranck,1788) Madsen,1949
}

\section{Rozeverter Moreno Fernandes ${ }^{1}$ Maria de Lurdes de Azevedo Rodrigues ${ }^{2}$ Hélcio Resende Borba $^{3}$ Maria Zenaide de Lima Chagas Moreno Fernandes ${ }^{4}$ Alziro de Amorim 5}

\begin{abstract}
Estudou-se a atividade anti-helmíntica das plantas; Allium sativum, Punica granatum, Tynnanthus fasciculatus e Cocos nucifera. Foram utilizados 70 frangos infectados naturalmente com Heterakis gallinarum, divididos em grupos de dez animais, com um controle positivo (20) $e$ um negativo (10). Administraram-se as plantas na forma de extrato aquoso e suco por gavage e trituradas incorporadas à ração nas doses de 2, 3 e $10 \mathrm{~g} \mathrm{~kg}^{-1} \mathrm{dia}^{-1}$, durante três dias consecutivos. Os resultados foram avaliados através de teste não. O A. sativum, P. granatum, T. fasciculatus $\boldsymbol{e}$ C. nucifera eliminaram: 6,$70 ; 4,12 ; 1,25$ e $0,22 \%$, respectivamente. Os percentuais de eliminação do A. sativum e do T. fasciculatus, corresponderam respectivamente a $1 / 4$ e $1 / 6$ do controle positivo, sendo o $\boldsymbol{C}$. nucifera e o $\boldsymbol{P}$. granatum semelhantes ao controle negativo. Desta forma, as plantas nas doses empregadas não apresentaram atividade significativa $(P<0,05)$ sobre o helminto $\boldsymbol{H}$. gallinarum .
\end{abstract}

Palavras-chave: Heterakis gallinarum, atividade antihelmíntica, plantas medicinais e frango.

\section{ABSTRACT}

The anthelminthic activity of the plants; Allium sativum, Punica granatum, Tynnanthus fasciculatus and Cocus nucifera was studied. Seventy chickens were used, naturally infected with Heterakis gallinarum, divided in groups of ten, with a positive control (20) and a negative (10). The plants were administered in aqueous extract and juice for gavage, and triturated, incorporate to the ration in the dosis of 2, 3 and $10 \mathrm{~g} / \mathrm{kg} /$ day, for three consecutive days. The results were appraised through no-parametric test. A. sativum, P. granatum, T. fasciculatus and C. nucifera eliminated: $6.70 ; 4.12 ; 1.25$ and $0.22 \%$, respectively. The percentile of elimination of $\boldsymbol{A}$. sativum and $\boldsymbol{T}$. fasciculatus, corresponded respectively to $1 / 4$ and 1/6 of the positive control, being $\boldsymbol{C}$. nucifera and $\boldsymbol{P}$. granatum similar to negative control. This way, the plants at applied doses didn't present significant activity $(P<0.05)$ on the helmint $\boldsymbol{H}$. gallinarum.

Key words: Heterakis gallinarum, anthelminthic activity, medicinal plants and chicken.

A produção de frangos e ovos com a utilização de sistemas orgânicos e outros não intensivos vem crescendo em importância em vários países. Em contraste com o sistema convencional, estas criações são afetadas por várias mudanças no manejo, tais como: acesso a áreas de pasto e proibição no uso de medicamentos preventivos, incluindo antiparasitários. Tal modelo implica o aperfeiçoamento do manejo e alimentação das aves, uma vez que tendo acesso a piquetes as aves terão contato com ovos, larvas e hospedeiros intermediários de diversos helmintos (TRAMSBORG et al., 1999). Assim sendo, objetivou-se com este trabalho determinar a atividade anti-helmíntica de plantas em frangos naturalmente infectados com $\boldsymbol{H}$. gallinarum.

Neste estudo, foram utilizados 70 frangos da marca Hubbard, ambos os sexos, com cerca de $3.300 \mathrm{~g}$, infectados naturalmente com $\boldsymbol{H}$. gallinarum.

\footnotetext{
${ }^{1}$ Médico Veterinário, Doutor, Professor Adjunto 4, Departamento de Morfofisiologia Veterinária (DMV), Centro de Ciências Agrárias (CCA), Universidade Federal do Piauí (UFPI), Campus Agrários da Socopo, CCA, S/N, 64.049-550. E-mail: zmoreno@ufpi.br. ${ }^{2}$ Médico Veterinário, Doutor, Profesora Adjunto, Departamento de Parasitologia Animal (DP), Instituto de Veterinária (IV), Universidade Federal Rural do Rio de Janeiro (UFRRJ).

${ }^{3}$ Biólogo, Doutor, Professor Adjunto, Departamento de Biologia Animal, Instituto de Biologia (IB), UFRRJ.

${ }^{4}$ Médico Veterinário, Mestranda do Curso de Ciência Animal da UFPI, CCA. E-mail: zenaidemoreno@bol.com.br

${ }^{5}$ Médico Veterinário, Doutor, Professor Titular Aposentado, Terapêutica e Toxicologia, UFRRJ.
} 
Constituiu-se sete grupos contendo dez animais, sendo quatro grupos teste, dois controle negativo para verificação da eliminação espontânea e um controle positivo tratados com mebendazol, incorporado à ração na dose de $0,031 \mathrm{~g} / \mathrm{kg} / \mathrm{dia}$. Os animais foram colocados em gaiolas, com um período de adaptação de 72 horas, recebendo diariamente $100 \mathrm{~g}$ de ração e água $\boldsymbol{a d}$ libitum. Antes da administração as aves ficaram em jejum de 18 horas com água à vontade.

A matéria vegetal foi administrada durante três dias consecutivos de acordo com a indicação popular (Figura 1). Após a administração, as fezes foram coletadas durante quatro dias consecutivos para cada grupo, em seguida foram lavadas e peneiradas em tamis de malhas 0,297 e 0,42mm e acondicionadas em frascos contendo uma solução a quente de ácido acético, formol e álcool (AFA), visando à contagem dos helmintos eliminados. No quinto dia de tratamento, os frangos foram sacrificados e necropsiados para colheita do conteúdo gastro-intestinal para contagem e identificação dos helmintos remanescentes.

A eficácia do tratamento foi avaliada pelo método crítico controlado (STEWARD, 1955) adaptado ao nosso modelo experimental. Os resultados foram expressos em termos de percentuais médios de nematóides eliminados nas fezes em relação ao total de helmintos recuperados na necropsia, e analisados estatisticamente através do teste não-paramétrico para comparação das médias por meio de valores de "U" (WHITNEY,1975).

Os resultados obtidos estão demonstrados na tabela 1: a eficácia do tratamento corresponde aos percentuais médios de eliminação fecal de $\boldsymbol{H}$. gallinarum. A prevalência observada foi de $100 \%$ para H. gallinarum; observou-se a presença de 99,2\% no ceco e $0,8 \%$ no cólon, o que concorda com VATNE
(1963) e PANKAVICH et al. (1973). A intensidade de infecção obtida nos testes superou o nível da infecção experimental descrita por PANKAVICH et al. (1973), permitindo a obtenção de resultados seguros quanto à eficácia das plantas e do mebendazol.

Nos animais tratados com extrato aquoso do alho, a planta apresentou uma discreta atividade em relação à $\boldsymbol{H}$. gallinarum, quando comparado ao grupo controle negativo (Tabela 1). Na figura 2, estão apresentados os percentuais acumulados de eliminação fecal de $\boldsymbol{H}$. gallinarum ao longo de 96 horas, comparado aos do anti-helmíntico padrão. O mebendazol apresentou um efeito gradativo em função da dose administrada, tornando-se expressivo no tempo de 72 horas $(24,7 \%)$. O efeito do alho tornou-se evidente em 48 horas, mantendo-se estável e situandose em $6,7 \%$, correspondendo a aproximadamente $1 / 4$ do efeito da droga padrão. KOBLOVA (1986), usando o mebendazol na dose $40 \mathrm{mg} / \mathrm{kg}$ em frangos naturalmente infectados com $\boldsymbol{H}$. gallinarum observou que a droga reduziu a infecção em 64\%. Observou-se ainda, eliminação tardia, tanto para o alho como para a droga padrão. Provavelmente, a localização cecal do nematóide dificultou o acesso dos agentes antihelmínticos, reduzindo sua ação. No entanto, o efeito do alho em termos de percentuais médios de eliminação foi sensivelmente superior ao do grupo controle e inferior ao mebendazol (Tabela 1).

O cipó-cravo incorporado à ração apresentou eficácia de aproximadamente 4,12\% (Figura 2) e seu efeito acumulado sobre a eliminação do $\boldsymbol{H}$. gallinarum, foi evidenciado 48 horas após a administração, com pico máximo em 72 horas. Este resultado, se comparado àquele obtido com a droga padrão, é aproximadamente seis vezes menor, sendo inferior aos descritos por AMORIM et al. (1991) em

\begin{tabular}{|l|l|l|c|c|c|}
\hline \multicolumn{1}{|c|}{ Familia } & \multicolumn{1}{|c|}{ Espéeie } & Nome Vulgar & $\begin{array}{c}\text { Parte } \\
\text { Usada }\end{array}$ & Estado & $\begin{array}{c}\text { Forma de Adm. e } \\
\text { Concentração }\end{array}$ \\
\hline Bignoniaceac & $\begin{array}{l}\text { Tynnanthus } \\
\text { fasciculatus Miers }\end{array}$ & Cipó-cravo & Caule & Seco & Ração $10 \%$ \\
\hline Liliaceae & Allium sativum L. & Alho & Bulbo & Fresco & Suco $10 \%$ \\
\hline Palmae & Cocos nucifera L.. & Coco-da-baia & Amêndoa & Maduro & Leite $100 \%$ \\
\hline Punicaceae & Punica granatum L. & Roma & Epicarpo & Seco & Ração $10 \%$ \\
\hline \hline
\end{tabular}

Figura 1 - Plantas utilizadas em testes de atividade anti-helmíntica em frangos naturalmente infectados com Heterakis gallinarum. 
Tabela 1 - Eficácia do alho, cipó-cravo, coco-da-baía, romã e do mebendazol em frangos naturalmente infectados com Heterakis gallinarum.

\begin{tabular}{|c|c|c|c|c|c|}
\hline & $\begin{array}{l}\text { Dose } \\
\text { g/kg/dia }\end{array}$ & $\begin{array}{l}\text { Número de helmintos } \\
\text { eliminados }\end{array}$ & $\begin{array}{l}\text { Contagem de helmintos } \\
\text { na necropsia (mín./máx.) }\end{array}$ & Total de helmintos & $\begin{array}{c}\text { Eliminação } \\
\text { fecal } \\
(\%)\end{array}$ \\
\hline Controle & 0,00 & 03 & $813(4-143)$ & 816 & 0,37 \\
\hline Alho & 2,00 & 36 & $503(22-145)$ & 539 & 6,70 \\
\hline Cipó-cravo & 3,00 & 22 & $511(15-99)$ & 533 & 4,12 \\
\hline Coco-da-baía & 10,00 & 03 & $237(5-87)$ & 240 & 1,25 \\
\hline Romã & 3,00 & 01 & $444(25-96)$ & 445 & 0,22 \\
\hline Mebendazol & 0,031 & 111 & $338(12-61)$ & 449 & $24,70 *$ \\
\hline
\end{tabular}

$*(\mathrm{P}<0,05)$.

testes conduzidos em camundongos naturalmente infectados com oxiurídeos.

O efeito acumulado do leite-de-coco na eliminação do $\boldsymbol{H}$. gallinarum nas primeiras 24 horas foi superior à droga padrão (Figura 2). No entanto, o resultado final obtido $(0,22 \%)$ é bem inferior ao relatado por AMORIM \&BORBA $(1994,1995)$ em camundongos naturalmente infectados com oxiurídeos. Quanto ao percentual de eliminação da romã, este foi inferior ao controle negativo (Figura 2), sendo pouco expressivos se comparados efeito anti-helmíntico registrado por AMORIM e BORBA (1990) em camundongos usando extratos da mesma. Desta forma, as plantas nas doses empregadas não apresentaram atividade significativa ( $\mathrm{P}<0,05)$ sobre o helminto $\boldsymbol{H}$. gallinarum .

\section{REFERÊNCIAS BIBLIOGRÁFICAS}

AMORIM, A.; BORBA, H.R. Ação anti-helmíntica de plantas III. Efeito de extrato aquoso de Punica granatum L. (romã) na eliminação de Vampirolepis nana e de oxiurídeos em camundongos. Rev Bras Farm, Rio de Janeiro, v.71, n.4, p.8587, 1990.

Ação anti-helmíntica de plantas X. Teste "in vivo" com extratos brutos de Cocos nucífera L. (Palmae). Rev Bras Farm, Rio de Janeiro, v.75, n.4, p.9192b, 1994.

Ação anti-helmíntica de Plantas XI. Influência de extratos brutos de Cocos nucifera L. (Palmae) na eliminação de Vampirolepis nana em camundongos. Rev Bras Farm, Rio de Janeiro v.76, n.4, p.98-99, 1995.

AMORIM, A.; BORBA, H.R.; AMANO, L.M. Ação antihelmíntica de plantas IV. Influência da casca do caule do cipó-cravo (Tynnantus fasciculatus, Miers, Bignoniaceae) na eliminação de Vampirolepis nana e oxiurídeos em camundongos. Rev Bras Farm, Rio de Janeiro, v.72, n.4, p.92-94, 1991.

KOBLOVA, I.A. Anthelmintics in experimental heterakiasis in

Figura 2 - Percentual de eliminação acumulada do Heterakis gallinarum em frango de corte tratados com o extrato aquoso do alho, cipó-cravo, coco-da-baía, romã e do mebendazol.

Ciência Rural, v.34, n.5, set-out, 2004. 
chickens. Helminthol Abst, (série A), v.55, n.6, p.242, 1986.

PANKAVICH, J.A. et al. Evaluation of Levamisole against experimental infections of Ascaridia, Heterakis and Capillaria spp. in chickens. Am J Vet Res, v.34, n.4, p.501-505, 1973.

STEWARD, J.S. Anthelmintic studies: I. A controlled critical entero-nemacidal test. Parasitol, v.45, p.231-241, 1955.

THAMSBORG, S.M.; ROEPSTORFF e LARSEN, M.
Integrated and biological control of parasites in organic and conventional production systems. Veterinary Parasitology, v. 84 n.3-4 p.169-186,1999.

VATNE, R.D. Aspects of the biology of Heterakis gallinarum (nematoda) in chicken and their host-parasite relations. Dissertation Abstract, v.24 n.4, p.1767-8, 1963.

WHITNEY, M. O caso de duas amostras independentes. In: SIEGEL, S. (Editor). Estatística não-paramétrica. McGrawHill do Brasil, 1975. 350p. p. 131-144. 